\title{
RELACIÓN ABUELO/A NIETO/A CUANDO EXISTEN CONFLICTOS FAMILIARES
}

\author{
Francisco Javier Balea-Fernández \\ Doctor en Psicología (USAL) \\ Doctorando en Investigación en Biomedicina (ULPGC) \\ fbalea@cop.es \\ Sonia González-Medina \\ Máster en Intervención y Mediación familiar, Social y Comunitaria (ULPGC) \\ songon78@hotmail.com \\ Javier Alonso-Ramírez \\ Doctorando en Investigación en Biomedicina (ULPGC) \\ xaalons086@gmail.com
}

Recepción Artículo: 18 enero 2020

Admisión Evaluación: 4 marzo 2020

Informe Evaluador 1: 13 marzo2020

Informe Evaluador 2: 18 marzo2020

Aprobación Publicación: 20 abril 2020

\section{RESUMEN}

En los últimos tiempos, la sociedad ha experimentado cambios, que ha llevado a analizar en profundidad las relaciones familiares, siendo la relación entre abuelos/as y nietos/as, la que ha causado mayor interés para los/as investigadores/as en las últimas décadas por la trascendencia que tienen los/as abuelos/as en la vida de sus nietos/as, creándose entre ellos/as una relación especial que no se establece con ningún otro miembro de la unidad familiar. La familia puede ser entendida como un conjunto o sistema estructurado, entre ellos existen lazos y relaciones afectivas, compartiendo metas, objetivos y proyectos comunes, creando una unidad que permite el reparto de funciones y responsabilidades entre sus miembros, por lo que un cambio en una de las partes repercute en las demás, y a consecuencia, de los cambios sufridos en la sociedad, los/as abuelos/as han adquirido una gran importancia en las relaciones familiares, concretamente en la relación con sus nietos/as. Con el fin de conocer las relaciones familiares especialmente entre abuelos/as y nietos/as, las dificultades de dicha relación ante situaciones de ruptura familiar, los derechos que ostentan los/as abuelos/as frente a sus nietos/as, y del conocimiento sobre los/as abuelos/as de los recursos existentes en materia de familias. Se ha llevado a cabo un estudio con diseño cualitativo fenomenológico a través de cuestionario en muestra de 46 participantes, abuelos/as de Gran Canaria, con edades comprendidas entre los 58 y 85 años un 67\% mujeres, y relación con hijo/as muy buena en el $54.3 \%$, buena $34.7 \%$ y regular $10.8 \%$. Se concluye que la principal dificultad para mantener una óptima relación entre abuelos/as y nietos/s está la separación/divorcio de sus hijos/as, por lo que se recurre en muchas ocasiones a la vía judicial.

Palabras clave: familia; abuelos; nietos; conflicto; derechos; recursos 


\section{RELACIÓN ABUELO/A NIETO/A CUANDO EXISTEN CONFLICTOS FAMILIARES}

\section{ABSTRACT}

Grandfather/grandchild relationship when there are family conflicts. In recent times, society has undergone changes, which have carried out an in-depth analysis of family relationships, being the relationship between grandparents and grandchildren the one that has been of most interest to researchers in recent decades due to its transcendence grandparents have in the lives of their grandchildren, creating a special relationship between them that is not established with any other member of the family unit. The family can be understood as a set or structured system, among them there are ties and affective relationships, sharing common goals, objectives and projects, creating a unit that allows the distribution of functions and controls among its members, making a change in a from the consequences it reverberates in the other consequences, the changes suffered in the society, the grandparents have acquired a great importance in the family relationships, specifically in the relationship with their grandchildren. In order to know family relationships, especially between grandparents and grandchildren, the difficulties of this relationship in situations of family breakdown, the rights of grandparents about their grandchildren and the knowledge of grandparents as well as the resources affected in terms of families. A study with a qualitative phenomenological design was carried out through a questionnaire in a sample of 46 participants, grandparents in Gran Canaria, aged between 58 and 85 years, with $67 \%$ of women, relationship with child at the most good at $54.3 \%$, good $34.7 \%$ and fair $10.8 \%$. It is concluded that the main difficulty to maintain an optimal relationship between grandparents and grandchildren is the separation/divorce of their children, which is why it is repeated on many occasions to the judicial route.

Keywords: family; grandparents; grandchildren; conflicto; rights; resources

\section{ANTECEDENTES}

La separación o divorcio son circunstancias que afectan a las relaciones familiares y sociales, que tienen una importante repercusión a nivel emocional. Esta circunstancia afecta a toda la familia con mayor impacto en la relación entre abuelas/os y nietas/os; de ello emana la Ley 42/2003, de 21 de noviembre, de modificación del Código Civil y de la Ley de Enjuiciamiento Civil en materia de relaciones familiares de los nietos con los abuelos, quien reconoce a los/as abuelos/as derechos y obligaciones frente a sus nietos/as, permitiéndoles seguir manteniendo la relación pese a la oposición de los/as progenitores/as y siempre que sea en beneficio del/la menor.

La evolución de la familia a lo largo de los siglos y a través de las culturas, ha suscitado diversas formas en la definición, no existiendo en la actualidad un único concepto ni una definición que describa todas las variedades tanto en composición como función o rol de cada componente. Una aproximación a este concepto puede ser considerada como la unión de personas, a las que les unen fuertes vínculos, objetivos y proyectos con unos mismos fines. Ampliando esta definición, los elementos comunes se estructuran en torno a un conjunto o sistema estructurado, teniendo en cuenta que entre ellos existen lazos y relaciones afectivas, compartiendo metas, objetivos y proyectos comunes, creando una unidad que permite el reparto de funciones y responsabilidades entre sus miembros. En definitiva, La familia es una totalidad, que aporta una realidad más allá de la suma de lo individual e incluso, más allá de los miembros que componen la familia. Esta totalidad se construye mediante un sistema de valores y creencias compartidos, por las experiencias vividas a lo largo de la vida, y por los rituales y costumbres que se transmiten generacionalmente (Espinal et al., 2006; López et al., 2014; Rodrigo y Palacios, 1998).

Las familias, tienen unas funciones en el marco de un escenario; se construyen personas adultas con una determinada autoestima y un determinado sentido de sí mismo y que experimentan un cierto nivel de bienestar psicológico en la vida cotidiana frente a los conflictos y situaciones estresantes; se preparan para aprender a afrontar retos, así como a asumir responsabilidades y compromisos que orientan a los adultos hacia una dimensión productiva, plena de realizaciones y proyectos e integrada en el medio social; lugar de encuentro intergeneracional donde los adultos amplían su horizonte vital formando un puente hacia el pasado (la generación de los abuelos) y hacia el futuro (la generación de los hijos); conforma una red de apoyo social para las diversas transiciones vitales; es fuente de apoyo instrumental, económico, informal y afectivo y la consecuencia que puede acarrear que surja cualquier problema o alejamiento entre adultos, es que se vea afectada la relación entre abuelos 
y nietos. Este escenario está enfocado a unas funciones como la educativa y de transmisión de valores, socialización y enlace de generaciones (Badenes y López, 2011; Prato et al., 2012; Rodrigo y Palacios, 1998).

La estructura familiar ha tolerado una gran transformación a lo largo del último siglo. Además de afectar a la estructura, ha modificado su ciclo y evolución, roles tradicionales y relaciones intergeneracionales. Las estructuras familiares más representativas son: familia extensa, nuclear, simultánea o reconstruida, monoparental y homoparental. En la figura 1 se muestran los porcentajes de estructuras de familias (Durán, 2017; Rondón y Munuera, 2009; Valdivia, 2008).

Figura 1: estructuras familias. Fuente: Durán (2017).

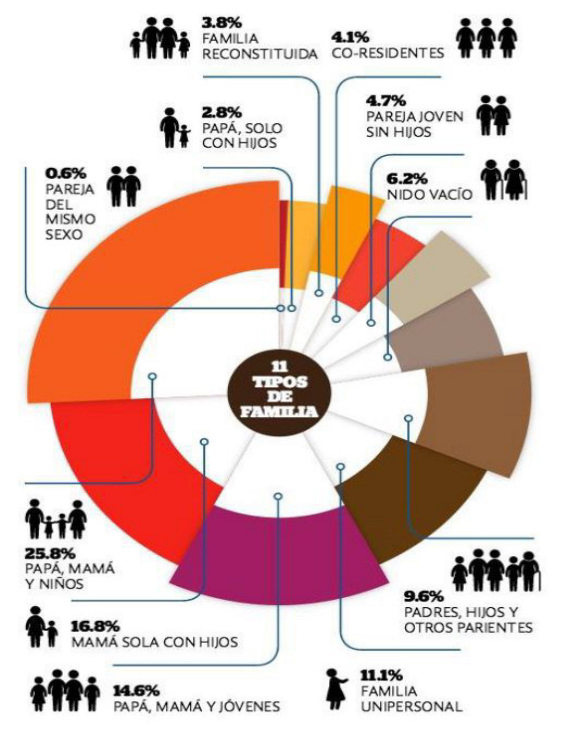

Por otro lado, además de las funciones y estructura familiar, se destacan las relaciones familiares intergeneracionales. Se han estudiado desde dos perspectivas: perspectiva sociológica: en estas relaciones se refleja la organización social, analizándose el valor de la influencia de cada generación en la sociedad y los recursos humanos y materiales que se pueden aportar y perspectiva psicológica: se ocupa de las relaciones intergeneracionales como una de las posibles formas de relación interpersonal (Castro, 2007).

Diversos estudios demuestran que cuando los/as abuelos/as mantienen una relación más cercana con sus hijos/as, ven con mayor frecuencia a sus nietos/as y como consecuencia, desarrollan un vínculo mayor con ellos. La generación de los padres es clave en la relación de los abuelos y los nietos. Por otro lado, la relación que se establece entre abuelos y nietos no puede medirse solamente por el número de contactos, sino que habría que tener también en cuenta la calidad de estos. La relación intergeneracional que se establece ocasiona un intercambio en las dos direcciones. Los abuelos dan a sus nietos cuidados, amor, valores morales, afecto, comprensión, experiencias de la vida, soporte, compañía, tiempo, amistad, y reciben de éstos estimulación, amor, entretenimiento, amistad, inspiración, compañía y continuidad en el futuro (Rico et al., 2001).

El rol del abuelo se muestra para la mayoría de los autores como un rol ambiguo y con escasa regulación social. Se identifican tres dimensiones del rol de abuelo: bienestar, significatividad o trascendencia y estilo, según los cuales se clarificaron cinco tipologías diferentes de ejercer la abuelidad (Noriega y Velasco, 2013): 


\section{RELACIÓN ABUELO/A NIETO/A CUANDO EXISTEN CONFLICTOS FAMILIARES}

1. Abuelos formales: son abuelos que asumen sus roles de forma tradicional y muestran un fuerte interés por sus nietos haciéndoles visitas, pero manteniendo un estilo rígido y autoritario.

2. Abuelo divertido: búsqueda de diversión, caracterizados por su tono informal, es un abuelo permisivo, relajado y jovial con sus nietos.

3. Abuelo distante: son abuelos autoritarios y distantes que apenas tienen contacto con sus nietos.

4. Abuelo de memoria histórica: abuelos guardianes de la memoria familiar.

5. Abuelos cuidadores/sustitutos (padres subrogados): son los abuelos que asumen la responsabilidad del cuidado y educación de los nietos durante gran parte del día.

Se entiende por separación o divorcio, la consecuencia de la decisión acordada entre los dos cónyuges o tan solo la voluntad de uno de ellos, por las diferencias irreconciliables que se produjeron en la pareja y esta decisión no sólo afecta a la individualidad sino también a todos los miembros de la familia, amigos y al círculo social en general. El proceso se caracteriza por cuatro momentos: separación (se instrumentaliza la ruptura, negociación); divorcio legal (desgaste emocional, sentimientos dicotómicos); divorcio emocional (elaboración del duelo) y post-divorcio (reequilibrio emocional, recuperación de la autonomía) (Fariña et al., 2005).

Tabla 1: Nulidades, separaciones y divorcios por CCEE en España. Fuente INE, (2017).

\begin{tabular}{|c|c|c|c|c|}
\hline & Total & Nulidades & Separaciones & Divorcios \\
\hline Total & 102.341 & 100 & 4.280 & 97.960 \\
\hline Andalucía & 18.295 & 19 & 840 & 17.437 \\
\hline Cataluña & 18.221 & 32 & 656 & 17.532 \\
\hline Madrid, Comunidad de & 14.668 & 18 & 541 & 14.109 \\
\hline Comunitat Valenciana & 11.864 & 14 & 523 & 11.327 \\
\hline Galicia & 5.614 & 1 & 193 & 5.419 \\
\hline Canarias & 5.308 & 0 & 200 & 5.108 \\
\hline Castilla y León & 4.078 & 4 & 207 & 3.868 \\
\hline País Vasco & 4.005 & 1 & 139 & 3.865 \\
\hline Castilla - La Mancha & 3.928 & 3 & 196 & 3.730 \\
\hline Murcia, Región de & 3.210 & 0 & 135 & 3.075 \\
\hline Balears, Illes & 2.694 & 0 & 146 & 2.548 \\
\hline Aragón & 2.389 & 1 & 101 & 2.286 \\
\hline Asturias, Principado de & 2.363 & 3 & 109 & 2.252 \\
\hline Extremadura & 1.940 & 2 & 114 & 1.824 \\
\hline Navarra, Comunidad Foral de & 1.414 & 0 & 64 & 1.350 \\
\hline Cantabria & 1.344 & 0 & 73 & 1.271 \\
\hline Rioja, La & 605 & 2 & 29 & 574 \\
\hline Ceuta & 216 & 0 & 4 & 212 \\
\hline Melilla & 186 & 0 & 13 & 172 \\
\hline
\end{tabular}

Las separaciones o divorcios tienen efectos negativos en el desarrollo intelectual, emocional y social de los/as menores, tras la separación de sus padres suelen experimentar una serie de intensas emociones y reacciones que varían en función a la edad de los niños. vienen asociadas a periodos de gran inestabilidad y desequilibrio en las relaciones familiares. Ello repercute en el clima que perciben los hijos en relación con el contexto familiar y escolar. Más que la separación en sí misma lo que afecta de manera más negativa al desarrollo de los niños, son las circunstancias que acompañan al proceso (Craig y Baucum, 1996).

El proceso de divorcio puede ser considerado un proceso de duelo por el que pasan todos los miembros de la estructura familiar. Las etapas del duelo se identificarán según las reacciones que provoca: fase de negación: no aceptar la noticia de divorcio; fase de dolor profundo: asumir las emociones negativas que se producen (sufrir pena y dolor emocional); fase de reajuste y adaptación: adaptarse a una nueva estructura familiar (conductas de carácter negativo: aislarse, no asumir las exigencias actuales; positivo: aprender a reajustar los roles y tareas, 
asumiendo lo que corresponde en cada momento); fase de cierre: recolocar emocionalmente el divorcio (William, 1997).

Desde el punto de vista normativo, la Ley 42/2003, 21 de noviembre de modificación del Código Civil y de la Ley de Enjuiciamiento Civil en materia de relaciones familiares de los nietos con los abuelos, como señala su exposición de motivos, prestar una mayor atención a las relaciones entre abuelos y nietos, dado que "Ios abuelos desempeñan un papel fundamental de cohesión y transmisión de valores en la familia", pudiendo quedar afectado dicho papel en los casos de situaciones de crisis matrimonial, de abandono de relaciones familiares no matrimoniales 0 de cumplimiento defectuoso de los deberes por parte de los progenitores y es que los abuelos "disponen de una autoridad moral y de una distancia con respecto a los problemas de la pareja que puede ayudar a los nietos a racionalizar situaciones de conflicto familiar, favoreciendo en este sentido su estabilidad y su desarrollo".

En resumen, se podría decir que el derecho a relacionarse, de visitas y de comunicación, variará dependiendo de la relación previa existente, en un entorno de desarrollo ordinario de una familia, en la que los abuelos y nietos mantienen una relación, no se puede entender que exista entre ellos un derecho a relacionarse, ya que dicha relación se produce de forma espontánea, por vínculos emocionales y afectivos. En cambio, cuando existe una causa que obstaculice 0 impida dicha relación, los abuelos podrán reclamar judicialmente el derecho de relación, de visitas y de comunicación, y es en estos casos, donde el juez deberá valorar la conveniencia o no de mantener dicha relación, velando siempre por los intereses del menor y teniendo en cuenta características como la edad del menor, la edad del abuelo, estado de salud del menor, estado de salud del abuelo, distancia entre domicilios, entre otras (Berrocal, 2005; Gracia, 2012).

\section{OBJETIVOS DE LA INVESTIGACIÓN}

Analizar desde la visión de los/as abuelos/as diferentes aspectos relacionados con las familias. El objetivo principal se amplía para determinar si conocen los recursos existentes en materia de conflictos familiares y la utilización de estos.

\section{MUESTRA Y/O PARTICIPANTES}

La muestra es de 46 abuelo/as del Centro de Día de Mayores de Telde (Canarias, España). Tras la oportuna autorización de los responsables del Centro, se procede a solicitar a los/as usuarios/as la participación en el estudio (requisito que tengan nietos/as). Todos los/as participantes del estudio, rellenaron el cuestionario de manera voluntaria y anónima, y se les trasladó que la privacidad de los datos obtenidos quedaba garantizada según lo dispuesto en la Ley Orgánica 3/2018, de 5 de diciembre, de protección de datos personales y garantía de los derechos digitales.

\section{METODOLOGía Y/O INSTRUMENTOS UTILIZADOS}

El diseño de esta investigación es observacional, descriptivo y transversal. El instrumento principal utilizado para la realización de este estudio es el cuestionario: ítems del 1 a 6: variables sociodemográficas (edad, el sexo, el nivel educativo alcanzado, el estado civil, el número de hijos/as y número de nietos/as); ́́tems del 7 al 13: variables de ámbito familiar (relación y dificultades, frecuencia, tiempo compartido mejora calidad de vida, hijos/as separados/as o divorciados/as); ítems del 14 al 17: variables institucionales (instituciones y/u organismos que ayuden a las familias ante un conflicto familiar).

\section{RESULTADOS ALCANZADOS}

\section{Resultados descriptivos:}

A continuación, se muestran los resultados descriptivos de la muestra por tipos de variables:

a.- Variables sociodemográficas:

Edad: media de edad: 70,93 (SD 7,36). 


\section{RELACIÓN ABUELO/A NIETO/A CUANDO EXISTEN CONFLICTOS FAMILIARES}

Sexo: mujer $65,21 \%$, hombre $34.79 \%$.

Nivel educativo: sin estudios: 19,56\%; EP: 45,66\%; ES: 28.26\%; superior: $6.17 \%$.

Estado civil: Casados/as: 50\%; Viudos/as: 30\%; separados/as: 18\%.

Número de hijos/as: media 2.91 (SD 4.90): $\leq 3$ hijos/as: 71.74\%; $\geq 4$ hijos/as: $28,26 \%$.

Número de nietos/as media 3,32 (SD 4,25): $\leq 4$ nietos/as: 73,93\%; $\geq 5$ nietos/as: 26,07\%.

\section{b.- Variables de ámbito familiar:}

Relación con los/as hijos/as: muy buena: $54,33 \%$; buena: $34,79 \%$; regular: $10,88 \%$.

Relación con nueras/yernos: muy buena: 30,43\%; buena: 36,96\%; regular: $32,61 \%$.

Relación con los/as nietos/as: muy buena: $67,4 \%$; buena: $26,09 \%$; regular: $6,51 \%$.

Frecuencia de visitas a nietos/as: nunca/vacaciones: 19,56\%; 1/mes: 4,34\%; 1/semana: 41,30\%; a diario: $34,78 \%$.

Compartir tiempo mejora la calidad de vida: mucho: $76,10 \%$; normal: $21,71 \%$; nada: $2,19 \%$.

Hijo/a divorciado/a: si: 69,56\%; no: 30,44\%.

Dificultad para relacionarse con nietos/as: si: 36,96\%; no: 63,04\%.

\section{c.- Variables institucionales:}

Abuela/o ha recurrido al ámbito jurídico: si: 69,57\%; no: 30,43\%.

Conoce alguna institución: si: 34,79\%; no: 65,21\%.

Acudiría en caso necesario a algún recurso: si: 82,6\%; 17,40\%.

Conoce la mediación familiar: si: 45,66\%; no: 54,34\%.

\section{Resultados por dimensiones:}

\section{a.- Relaciones familiares:}

Los/as abuelos/as mantienen muy buenas relaciones con sus nietos/as e hijos/as, lo que significa que mantener relaciones satisfactorias entre abuelos/as-padres/madres facilita las relaciones entre abuelos/as-nietos/as. En la relación establecida entre abuelos/as-nietos/as, uno de los factores que más influye, es la frecuencia de visitas, así se mejoran los vínculos familiares. Independientemente de la frecuencia de contactos y de los factores que influyen en dicha relación, los/as abuelos/as que comparten tiempo con sus nietos/as, consideran que aumenta la calidad de sus vidas.

\section{b.- Derechos de los abuelos/as:}

Un elevado porcentaje de abuelos/as consideraron que es necesario que se les consulte judicialmente durante el proceso separación o divorcio de sus hijas/os. Dicho derecho, está reconocido por el ordenamiento jurídico español como un derecho que tienen para relacionarse con sus nietos/as sin que pueda verse vulnerado salvo que exista justa causa. A pesar de que la ley entró en vigor hace casi dos décadas, algunos de los/as abuelos/as, manifiestan que, siguen teniendo dificultades para relacionarse con sus nietos/as.

\section{c.- Conocimiento de los recursos u organismos en materia de familias:}

Los/as abuelos/as, no conocen suficientemente los recursos existentes en materia de familias. La única institución que identificaron en relación con las familias es, Servicios Sociales. A pesar del desconocimiento en relación con las instituciones u organismos que prestan servicios en materia de familia. Se puede destacar que un porcentaje alto de los/as abuelos/as que participaron en este estudio, manifestaron que, en caso de necesitarlo, sí acudirían a alguno de los diferentes recursos existentes. Por último, en relación con si habían escuchado alguna vez hablar de Mediación Familiar, aunque las respuestas negativas obtuvieron un porcentaje un poco más alto que las respuestas afirmativas. De los/as abuelos/as que respondieron de manera positiva, describieron e identificaron la Mediación Familiar, como una ayuda a las familias.

\section{DISCUSIÓN}

Teniendo en cuenta que la familia es un conjunto o sistema estructurado, que entre ellos existen lazos y relaciones afectivas, compartiendo metas, objetivos y proyectos comunes, creando una unidad que permite el reparto 
de funciones y responsabilidades entre sus miembros, por lo que un cambio en una de las partes repercute en las demás y a consecuencia, de los cambios sufridos en la sociedad, los/as abuelos/as han adquirido una gran importancia en las relaciones familiares, concretamente en la relación con sus nietos.

Mencionando la dificultad de los/as abuelos/as para relacionarse con sus nietos/as cuando existe un conflicto familiar, tras analizar los datos obtenidos, se puede afirmar que las relaciones entre padres/madres-abuelos/as y entre abuelos/as-nietos/as, vienen marcadas por diferentes características que pueden influir en dichas relaciones. En líneas generales, se observa que las relaciones familiares existentes son buenas, destacando, sobre todo, la relación que mantienen los abuelos/as con sus nietos/as. Esta última, puede deberse a que las relaciones entre abuelos/as y nietos/as, vienen influenciadas por las relaciones entre padres/madres y abuelos/as. Es decir, si hay buena relación padre/madre-hijo/a, habrá buena relación abuelo/a-nieto/a. Rico, Serra y Viguer (2001), señalan que en múltiples investigaciones ha podido observarse como la generación de los padres es clave en la relación de abuelos/as y nietos/as. Por lo tanto, aquellos/as abuelos/as que mantienen una relación más cercana con sus hijos/as, ven con mayor frecuencia a sus nietos/as y como consecuencia desarrollan un vínculo mayor con ellos/as.

La relación entre abuelos/as y nietos/as, puede verse perjudicada por diversos conflictos familiares como una ruptura del matrimonio o pareja (separación o divorcio), el fallecimiento de uno de los progenitores o la existencia de diferencias personales entre los/as abuelos/as y los/as progenitores de los/as menores. En este estudio, nos hemos centrado en la dificultad de dicha relación tras una separación o divorcio y en referencia a los datos obtenidos, aproximadamente más de la mitad de la muestra, afirmó tener en la actualidad hijos/as separados/as 0 divorciados/as y aproximadamente la mitad de ese porcentaje afirma tener dificultades para relacionarse 0 visitar a sus nietos/as. De hecho, las separaciones vienen asociadas a periodos de gran inestabilidad y desequilibrio en las relaciones familiares. Ello repercute claramente en el clima que perciben los hijos en relación con el contexto familiar. Más que la separación en sí misma lo que afecta de manera más negativa al desarrollo de los niños, son las circunstancias que acompañan al proceso (Craig y Baucum, 1996). Los hijos experimentan la ruptura familiar en términos de pérdidas; de uno de los padres y de la familia extensa de este; de intercambios afectivos y comunicativos; de un modelo de identificación y orientación; del sentimiento de pertenencia a una familia, que le proporciona identidad, protección y seguridad (Tejedor y Jiménez, 2001).

Una vez producida la ruptura del matrimonio o pareja, los/as menores tienen derecho a seguir relacionándose y disfrutando de sus abuelos/as que bien puede quedar reflejado el régimen de visitas a través del convenio regulador, o bien los/as abuelos/as pueden reclamar dicho derecho a relacionarse con sus nietos cuando realmente se les esté privando de ello. Por lo tanto, es un aspecto para resaltar en este estudio, el desconocimiento de más de la mitad de los/as abuelos/as sobre los recursos y organismos existentes en materia de ayuda a las familias y el porcentaje tan alto de estos abuelos/as que confirman que acudirían a estos recursos en caso necesario. Sobre todo, si tenemos en cuenta que la Ley 42/2003, entró en vigor hace casi dos décadas dando respuesta a que los/as abuelos/as tuvieran derecho de relación, de visitas y de comunicación.

\section{CONCLUSIONES}

Haciendo una síntesis de todo lo expuesto, se puede concluir en líneas generales que las relaciones entre abuelos/as-nietos/as es un campo de estudio reciente y existe poca literatura científica al respecto, siendo importante el seguir investigando en dicha materia de estudio.

Las relaciones entre abuelos/as y nietos/as, son imprescindibles para ambos, creándose entre ellos/as un vínculo especial de reciprocidad, aportando cada uno/a aspectos fundamentales al otro/a; además, en la relación entre abuelos/as y nietos/as, no sólo importa la frecuencia, sino la calidad del tiempo que comparten juntos/as.

La relación que los/as padres/madres mantienen con los/as abuelos/as, influye en la relación entre los/as abuelos/as y los/as nietos/as, ya que son ellos/as los mediadores dentro de las relaciones, pudiendo facilitar 0 dificultar dicha relación. Por otro lado, una de las dificultades en la relación entre abuelos/as y nietos/as, es cuan- 


\section{RELACIÓN ABUELO/A NIETO/A CUANDO EXISTEN CONFLICTOS FAMILIARES}

do se produce en la familia una situación de separación o divorcio, siendo este, uno de los factores determinantes en las relaciones entre abuelos/as y nietos/as.

El medio más utilizado por los/as abuelos/as cuando exista una causa que obstaculice o impida la relación es la reclamación judicial del derecho de relación, de visitas y de comunicación.

\section{REFERENCIAS BIBLIOGRÁFICAS}

Badenes, N., y López, M. T. (2011). Doble dependencia: abuelos que cuidan nietos en España. Revista Zerbitzuan, 49, 107-125.

Berrocal, A. I. (2005). Reflexiones sobre las relaciones familiares entre abuelos y nietos tras la nueva Ley 42/2003, de 21 de noviembre. Anuario de Derechos Humanos, 6, 11-112.

Castro, M. (2007). Relaciones intergeneracionales y bienestar de las personas mayores [Universidad de Granana]. http://digibug.ugr.es/bitstream/10481/1634/1/1684256x.pdf

Craig, G., y Baucum, D. (1996). Desarrollo psicológico (Pearson Ed).

Durán, E. (2017). Los distintos tipos de familia que existen y sus características. http://wradio.com.mx/programa/2017/01/17/en_buena_onda/1484617501_114526.html

Espinal, I., Gimeno, A., y González, F. (2006). El enfoque sistémico en los estudios sobre la familia. Revista Internacional de Sistemas, 14, 21-34.

Fariña, F., Tortosa, F., y Arce, R. (2005). Psicología jurídica del menor y el contexto familiar: Una mirada al pasado, presente y futuro. Psicología Jurídica Del Menor y de La Familia, 9-27.

Gracia, J. (2012). El derecho a las relaciones personales entre los nietos y sus abuelos. Una aproximación sociojurídica. Revista Electrónica de Derecho de La Universidad de La Rioja, 10, 105-122.

INE. (2017). Nulidades, separaciones y divorcios por Comunidades Autónomas. https://www.ine.es/jaxiT3/Tabla.htm?t=20172

López, J., Pérez-Rojo, G., Noriega, C., y Velasco, C. (2014). Ser abuelos hoy. Relaciones intergeneracionales en la familia (Dykinson).

Noriega, C., y Velasco, C. (2013). Relaciones abuelos-nietos: una aproximación al rol de abuelo. Revista de Ciencias Sociales, 41, 464-482.

Prato, A., Hernández, A. bL., Techera, L., y Rivas, R. (2012). Abuelos y nietos ¿una relación necesaria? Revista de Biomedicina, 7(2), 22-36.

Rico, C., Serrra, E., y Viguer, P. (2001). Abuelos y nietos. Abuelo favorito, abuelo útil (Pirámide).

Rodrigo, M. J., y Palacios, J. (1998). Conceptos y dimensiones en análisis evolutivo-educativo de la familia, en Familia y desarrollo humano (Alianza).

Rondón, L. M., y Munuera, M. P. (2009). Mediación familiar: un espacio de intervención para trabajadores sociales. Revista de Trabajo Social, 11, 25-41.

Tejedor, A., y Jiménez, F. (2001). Evaluación psicológica en los supuestos de guarda y custodia (Jiménez, F). Valdivia, C. (2008). La familia: concepto, cambios y nuevos modelos. Revista La Revue Du REFIF, 1, 12-22. William, J. (1997). El tratamiento del duelo: asesoramiento psicológico y terapia (Paidós). 